\title{
Tumor diversity and evolution revealed through RADseq
}

\author{
Elizabeth B. Perry ${ }^{1,2}$, Alvin Makohon-Moore ${ }^{3}$, Caihong Zheng ${ }^{4}$, Charles K. Kaufman ${ }^{5}$, \\ Jun $\mathrm{Cai}^{4}$, Christine A. Iacobuzio-Donahue ${ }^{3}$ and Richard M. White ${ }^{1}$ \\ ${ }^{1}$ Cancer Biology \& Genetics, Memorial Sloan Kettering Cancer Center, New York, New York, USA \\ 2 Biostatistics, Yale University, New Haven, Connecticut, USA \\ ${ }^{3}$ The David M. Rubenstein Center for Pancreatic Cancer Research, Human Oncology and Pathogenesis Program, Memorial \\ Sloan Kettering Cancer Center, New York, New York, USA \\ ${ }^{4}$ Key Laboratory of Genomic and Precision Medicine, Beijing Institute of Genomics, Chinese Academy of Sciences, University \\ of Chinese Academy of Sciences, Beijing, China \\ ${ }^{5}$ Washington University School of Medicine, St. Louis, Missouri, USA \\ Correspondence to: Elizabeth B. Perry, email: ElizabethBPerry@gmail.com
}

Richard M. White, email: whiter@mskcc.org

Keywords: restriction-site associated DNA sequencing, RADseq, cancer, next-generation sequencing, tumor evolution $\begin{array}{lll}\text { Received: April 09, } 2017 \quad \text { Accepted: May 12, } 2017 & \text { Published: June 03, } 2017\end{array}$

Copyright: Perry et al. This is an open-access article distributed under the terms of the Creative Commons Attribution License 3.0 (CC BY 3.0), which permits unrestricted use, distribution, and reproduction in any medium, provided the original author and source are credited.

\section{ABSTRACT}

Summary: Cancer is an evolutionary disease, and there is increasing interest in applying tools from evolutionary biology to understand cancer progression. Restriction-site associated DNA sequencing (RADseq) was developed for the field of evolutionary genetics to study adaptation and identify evolutionary relationships among populations. Here we apply RADseq to study tumor evolution, which allows for unbiased sampling of any desired frequency of the genome, overcoming the selection bias and cost limitations inherent to exome or whole-genome sequencing. We apply RADseq to both human pancreatic cancer and zebrafish melanoma samples. Using either a low-frequency (SbfI, $0.4 \%$ of the genome) or high-frequency (NsiI, $6-9 \%$ of the genome) cutter, we successfully identify single nucleotide substitutions and copy number alterations in tumors, which can be augmented by performing RADseq on sublineages within the tumor. We are able to infer phylogenetic relationships between primary tumors and metastases. These same methods can be used to identify somatic mosaicism in seemingly normal, non-cancerous tissues. Evolutionary studies of cancer that focus on rates of tumor evolution and evolutionary relationships among tumor lineages will benefit from the flexibility and efficiency of restriction-site associated DNA sequencing.

\section{INTRODUCTION}

The characterization of cancer as an evolutionary process was reviewed by Peter Nowell four decades ago. He hypothesized a stepwise progression of acquired variation and natural selection to explain the emergence and increasing aggressiveness of tumors [1]. Since then, data from cancer genomics, multi-region sequencing, and single-cell sequencing have provided new insight into the complex clonal evolution of tumors and the extensive genetic heterogeneity present within and between cancer patients. The mechanisms underlying the generation and maintenance of tumor diversity are of particular interest to those seeking to understand disease progression and therapeutic resistance of advanced cancers. A multidisciplinary approach has been increasingly applied to problems of clonal evolution in cancer [2-6]. Theory and tools from evolutionary biology have been applied to the field of cancer biology to understand the rates of accumulation of mutations in cellular lineages [5,7], spatial and temporal patterns of intratumor heterogeneity [8-10], the timing and order of metastatic progression [11-14], and optimal strategies for therapeutic dosing and schedules [15-17]. However, 
a large disciplinary divide still exists between cancer biology and evolutionary biology, and potentially useful theoretical and experimental tools have yet to be applied across disciplines.

Restriction-site associated DNA sequencing (RADSeq) was initially developed for studies of evolutionary genetics and has proven to be a powerful tool in this field. This method has been largely overlooked in cancer biology, which instead relies almost exclusively on whole-genome sequencing and exome sequencing to discover and quantify genetic variation in tumors (see Figure 1 for a comparison of the three methods). Like exome sequencing, RADSeq is a reduced-representation sequencing approach that targets a subset of the genome. However, instead of targeting exonic regions for sequencing, the RADseq protocol targets regions of the genome flanking restriction enzyme cut sites. Several variations of RADseq have been described [18-24]. In brief, high quality genomic DNA is first digested with a restriction enzyme. Then, sequencing adapters (doublestranded oligos compatible with a next generation sequencing platform) are ligated onto the characteristic sticky ends generated by the restriction enzyme digestion. The highly-specific ligation of sequencing adapters to digested cut sites allows for the targeted sequencing of regions flanking these positions, therefore no capture step is required. Barcode sequences can also be included in the adapter oligos to allow for multiplexed sequencing of multiple samples in the same sequencing lane.

RADseq is a highly flexible approach because the proportion of the genome targeted for sequencing can be controlled through the choice of restriction enzyme. Common cutters (typically enzymes with a shorter recognition sequence,) will target a higher percentage of genome for sequencing than rare cutters. This means that common cutters can be used for questions that require more sequence information per genome, for example to distinguish between recently-diverged populations (e.g. primary tumors vs. metastases) or tumors with low mutation rates/burdens (e.g. pediatric tumors). Rare cutters can be selected for research questions that call for fewer sites per genome and a greater depth of sequencing per site (e.g. heterogeneity questions), or benefit from a large number of samples (e.g. many tumor sites or patients).

RADseq differs from whole-exome sequencing in that it does not specifically target functional regions of the genome. Restriction cut sites occur throughout the genome, largely without bias, and thus the sequenced regions will represent coding DNA as well as many intergenic and other non-coding sites where non-coding RNAs might be transcribed as well. This important distinction means that RADseq will likely not be the most efficient method to identify driver mutations and protein changes responsible for the cancer phenotype. However, RADseq does have the potential to be a more useful tool than exome sequencing to study tumor phylogenetics and intrinsic mutation rates, for which neutrally evolving sites provide the most robust information [25-27]. These differing strengths make whole-exome sequencing and RADseq complimentary methods for a variety of cancer biology questions. Here we test the utility of RADseq for cancer genomics by applying RADseq to a zebrafish model of melanoma and to human pancreatic tumors.

\section{RESULTS}

\section{Transgenic zebrafish model of melanoma}

\section{Performance}

We applied RADseq to a zebrafish model of melanoma to identify mutations from matched pairs of tumor and normal tissue (Figure 2A). Transgenic p53-deficient zebrafish expressing the mutant form of human $B R A F(V 600 E)$ in melanocytes spontaneously develop melanoma at 4-12 months of age ; $\operatorname{Tg}$ (mitfa:BRAF(V600E)); $p 53^{-/}[28,29]$. We dissected melanoma tumors and normal skin from three adult fish and used the restriction enzyme SbfI (a rare cutter, with an $8 \mathrm{bp}$ recognition sequence) to prepare RADseq libraries for sequencing on the Illumina platform (HiSeq2500). In silico analysis using the zebrafish reference genome [30] predicts 30,667 SbfI restriction enzyme cut sites in the genome. Because the recognition sequence of the restriction enzyme is palindromic, each occurrence of the recognition sequence results in two cuts, one on each opposing DNA strand. This generates sequence coverage in both directions from the cut site $[22,23]$. We sequenced $100 \mathrm{bp}$ flanking both sides of each cut site in the $1.4 \mathrm{~Gb}$ genome, resulting in representation of approximately $0.4 \%$ of the genome. The average depth of coverage for these loci was $\sim 350 \mathrm{x}$, and coverage was even across loci (358x mean, 337x median).

After filtering reads for quality, we found that $91.5 \%$ of sequencing reads successfully mapped to the zebrafish reference genome and $94.2 \%$ of these reads mapped specifically to the predicted cut site regions. This degree of specificity is striking, especially considering the high genetic divergence between different experimental lines of zebrafish, including differences between our lab strains and the sequenced reference line that we used to predict cut sites [31, 32].

\section{Mutation-calling in tumors}

RADseq data can be analyzed using standard tools for alignment to a reference genome and genotyping/ mutation calling [19]. We aligned reads to the zebrafish (danRer7) reference genome using BWA [33] and identified mutations with MuTec [34] (see methods). By comparing melanoma tumor to normal skin for each adult fish, we were able to identify single nucleotide variants 
(SNVs). The total number of SNVs identified in the three adult fish tumors were 3, 8, and 5 (Figure 2B). These numbers are consistent with previous characterization of engineered zebrafish melanomas from whole exome sequencing which capture $\sim 3 \%$ of the genome and find low mutational burden on average, with significant variation among tumors [35].

\section{Restriction enzyme selection}

To demonstrate how the choice of restriction enzyme influences the number of mutations captured with RADseq, we used the same genomic DNA from one of the SbfI-digested fish described above and made an additional RADseq library using a more frequentlycutting enzyme, NsiI (a 6bp recognition sequence). In silico analysis predicts 687,595 NsiI cut sites in the

A

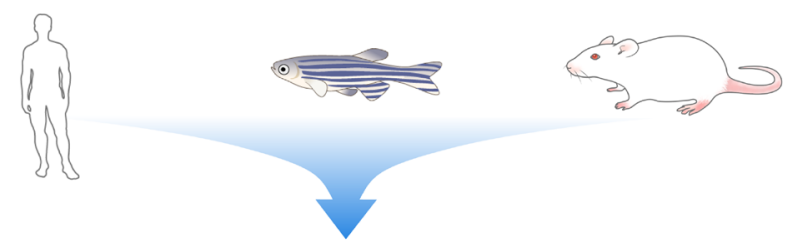

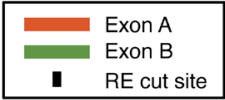

Whole-genome sequencing

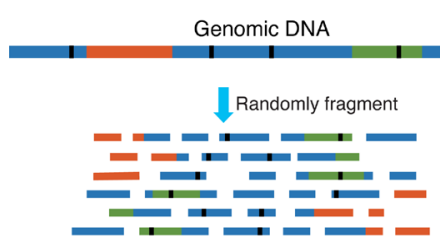

Ligate sequencing adaptors

\begin{tabular}{llll}
\hline P1 & $P 2$ & $P 1$ & $P 2$
\end{tabular}

$P 1, P 2$

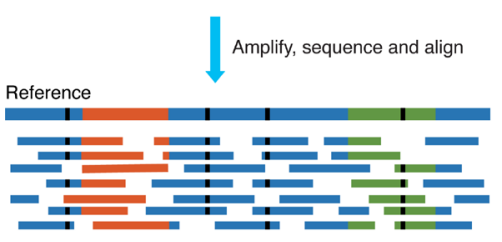

Exome sequencing

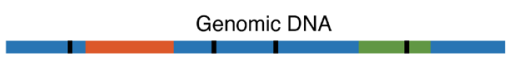

Genomic DNA

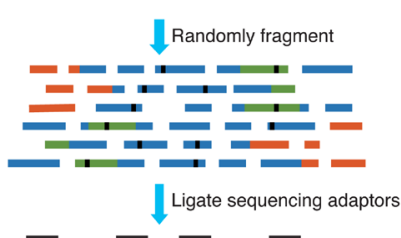

\begin{tabular}{lllll}
\hline $\mathrm{P} 1$ & $\mathrm{P} 2$ & $\mathrm{P} 1$ & $\mathrm{P} 2$ \\
\hline
\end{tabular}

P1 - P 2

Target capture \& Wash

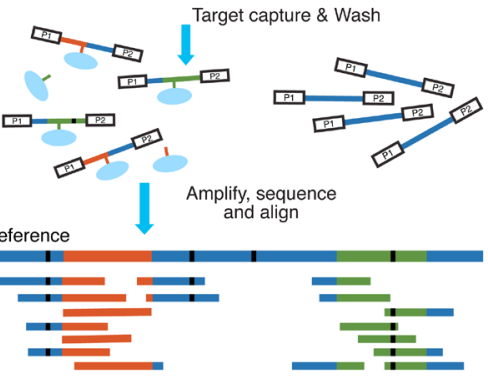

RADseq

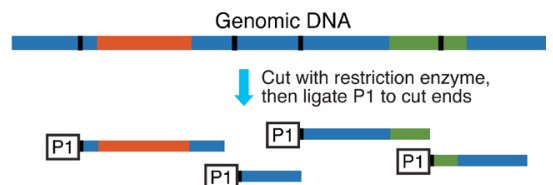

P1

Randomly fragment

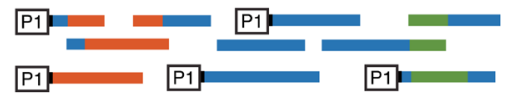

Ligate P2

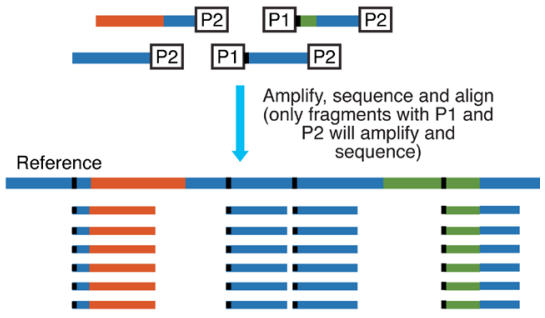

B

\begin{tabular}{llll}
\hline & \multicolumn{1}{c}{ WGS } & WES & RAD \\
\hline $\begin{array}{l}\text { Regions of } \\
\text { genome targeted }\end{array}$ & $\begin{array}{l}\text { All accessible• regions of the } \\
\text { genome; genes and intergenic }\end{array}$ & $\begin{array}{l}\text { Representation determined by the } \\
\text { capture kit; enriching for exons and UTRs }\end{array}$ & $\begin{array}{l}\text { Largely unbiased representation of } \\
\text { genome; genes and intergenic }\end{array}$ \\
\hline $\begin{array}{l}\text { Percent genome } \\
\text { represented }\end{array}$ & Fixed, typically 90\% genome & Fixed, typically 2\% genome & Flexible, typically 0.1\% 10\% genome \\
\hline $\begin{array}{l}\text { Species specific } \\
\text { reagents }\end{array}$ & $\begin{array}{l}\text { Same reagents can be } \\
\text { used across species }\end{array}$ & Species-specific capture step & Same reagents can be used across species \\
\hline
\end{tabular}

Figure 1: A. Comparison of whole-genome, exome and RADseq approaches to cancer genome. Whereas whole genome sequencing allows for an unbiased view of the genome, it requires high cost when high sequencing depth is needed. Exome sequencing is a reduced representation approach that is cost-effective but gives a highly biased view of only protein coding genes, which are under selection in many cancers. RADseq uses restriction sites naturally dispersed across the genome at both intergenic as well as coding regions, combining the benefits of whole-genome and exome sequencing to allow for unbiased, high sequencing depth in a highly cost effective manner. B. Specific features of the 3 methods as they apply to cancer biology. 
A

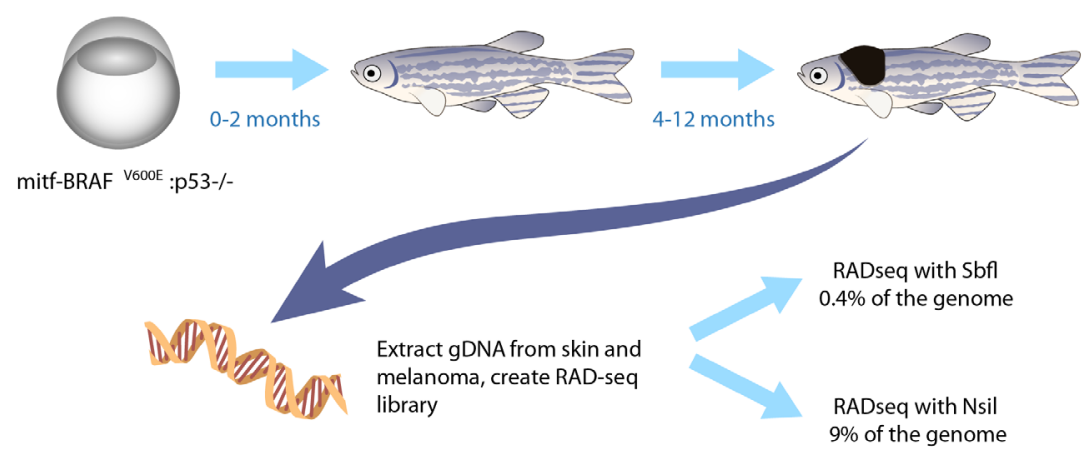

B

\begin{tabular}{cccccc}
\hline Sample ID & $\begin{array}{c}\text { Restriction } \\
\text { enzyme }\end{array}$ & $\begin{array}{c}\text { Percent genome } \\
\text { represented }\end{array}$ & $\begin{array}{c}\text { \# predicted sites in genome } \\
\text { (\% on target in seq) }\end{array}$ & $\begin{array}{c}\text { Sequencing } \\
\text { depth }\end{array}$ & $\begin{array}{c}\text { \# mutations } \\
\text { tumor vs. skin }\end{array}$ \\
\hline BRAF; p53 fish \#1 & Sbfl & 0.4 & $\begin{array}{c}30,667 \\
(94 \% \text { on target) }\end{array}$ & $358 \times$ & 3 \\
\hline BRAF; p53 fish \#2 & Sbfl & 0.4 & $\begin{array}{c}30,667 \\
(97 \% \text { on target) }\end{array}$ & $87 \times$ & 8 \\
\hline BRAF; p53 fish \#3 & Sbfl & 0.4 & $\begin{array}{c}30,667 \\
(98 \% \text { on target) }\end{array}$ & $49 x$ & 5 \\
\hline BRAF; p53 fish \#3 & Nsil & 9 & $\begin{array}{c}687,595 \\
(98 \% \text { on target) }\end{array}$ & $36 x$ & 47 \\
\hline
\end{tabular}

c
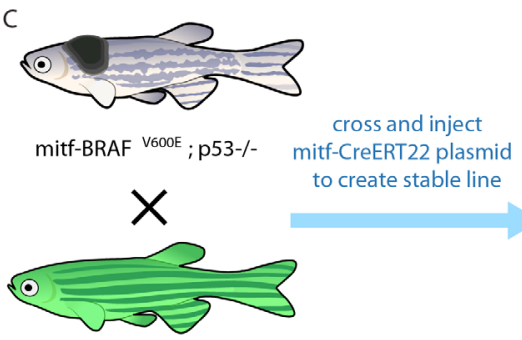

ubb-lox-GFP-lox-mCherry

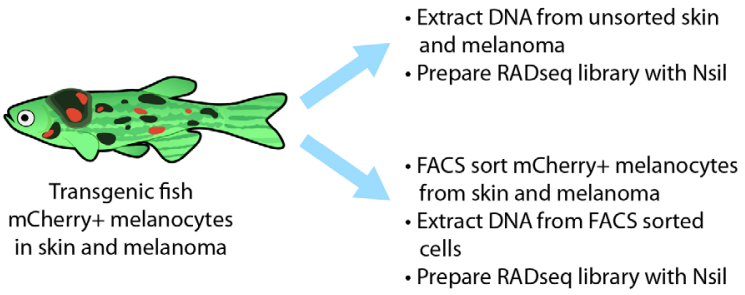

D

Variant allele fraction: overall

Variant allele fraction: specific loci
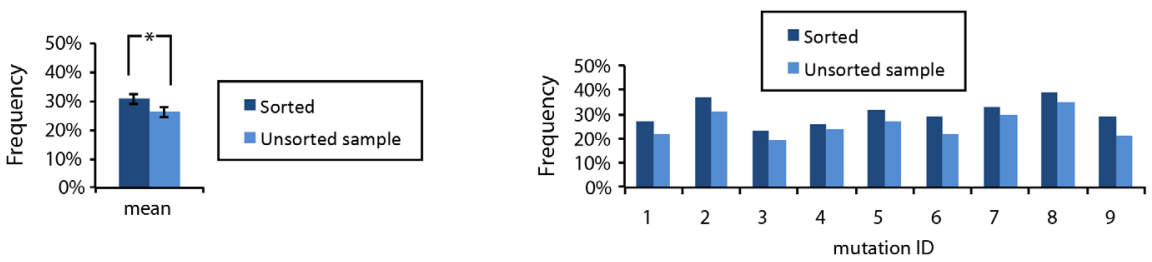

Figure 2: Applying RADseq to a zebrafish model of melanoma. A. A transgenic zebrafish model in which the mitfa promoter drives human oncogenic BRAFV600E. The embryos (left) and young adults (center) are relatively normal, but all adults (right) develop clinically overt melanomas within 4-12 months. Genomic DNA was isolated from a melanoma as well as surrounding skin in four of these transgenic animals and RADseq libraries were created from gDNA using either SbfI ( $0.4 \%$ of the genome) or NsiI ( $9 \%$ of the genome). B. The efficiency of reads mapping to restriction enzyme cut sites in the genome, along with the depth of coverage and the number of mutations discovered in each melanoma. We found a small number of mutations in 3 melanomas when sampling $0.4 \%$ of the genome. For one fish, the same genomic DNA was used for RADseq with NsiI, and shows a much higher number of mutations, given the greater coverage of the genome. C. A fate-mapping transgenic line was created to assess the efficiency of RADseq on subpopulations of cells. The mitf-BRAF;p53 model was bred with a fluorescent color "switch" line in which a floxed GFP cassette can be swapped for an mCherry cassette upon Cre-mediated recombination. This cross was injected with a melanocyte-specific mitf-CreERT2 plasmid. Upon endoxifen or tamoxifen treatment, a subset of the melanocytes in the skin as well as within the melanoma switched from GFP to mCherry. Genomic DNA was extracted from either the bulk tumor/skin, or from FACS sorted mCherry+ melanocytes from the tumor/skin pair. D. Overall variant allele fractions across all mutations in the sorted $v s$. unsorted populations showed a significant increase in the mCherry + subpopulations $\left({ }^{*}, p<0.05, t\right.$-test $)$. E. Individual mutation variant allele fractions are shown, consistently demonstrating increased sensitivity in the sorted population, which facilitates higher-confidence mutation calling in subclonal populations. 
zebrafish genome, and again we found our reads mapped to these regions with an on-target rate greater than $90 \%$. Sequencing $100 \mathrm{bp}$ flanking each side of these cut sites results in representation of approximately $9 \%$ of the zebrafish genome. As expected, with many more bases in the genome represented in the NsiI library, the number of mutations was greater than for the SbfI library - we detected 47 mutations in NsiI-flanking regions versus the 5 mutations detected in the SbfI-flanking regions of the same melanoma tumor (Figure 2B and Supplemental Table 1).

\section{Lineage-tracing model to study tumor evolution of subclonal populations}

One of the challenges to studying genetic evolution in tumors is heterogeneity - multiple subclonal tumor lineages typically make up a single biopsy or tissue sample [36]. In addition, normal cells such as fibroblasts and immune cells are present within a dissected tumor sample, at varying frequencies [37]. This means that typical libraries prepared for tumor sequencing represent a mixture of cells with different genotypes. Single-cell sequencing has promise to overcome some of these challenges, but it remains technically difficult and expensive to characterize comprehensive mutational profiles from single cells [38].

We developed a transgenic zebrafish with an inducible system to selectively trace subsets of melanocytes both in normal skin as well as within the melanoma (Figure 2C). We started with an existing zebrafish line (ubi:Switch) [39, 40] that possesses a ubi:loxP- GFP-loxP-mCherry cassette. These

A

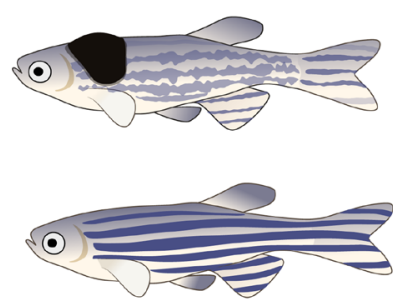

B

\begin{tabular}{llccc}
\hline Genotype & Phenotype & $\begin{array}{c}\text { Restriction } \\
\text { enzyme }\end{array}$ & Tissue & \# mutations \\
\hline BRAF; p53 & Melanoma-prone & Sbfl & Brain & 1 \\
\hline BRAF; p53 & Melanoma-prone & Sbfl & Skin & 0 \\
\hline BRAF; p53 & Melanoma-prone & Sbfl & Melanomatumor & 3 \\
\hline BRAF; p53 & Melanoma-prone & Sbfl & Liver & 0 \\
\hline
\end{tabular}

D

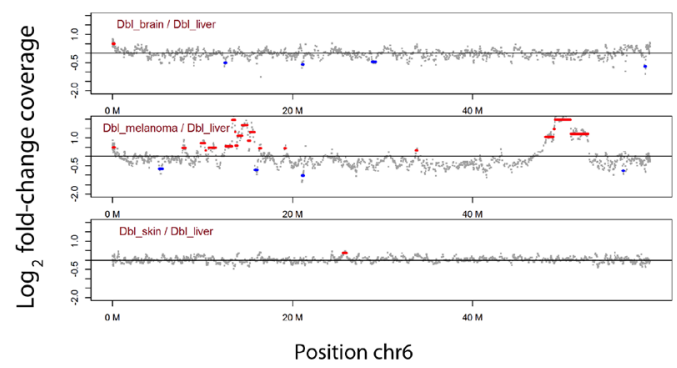

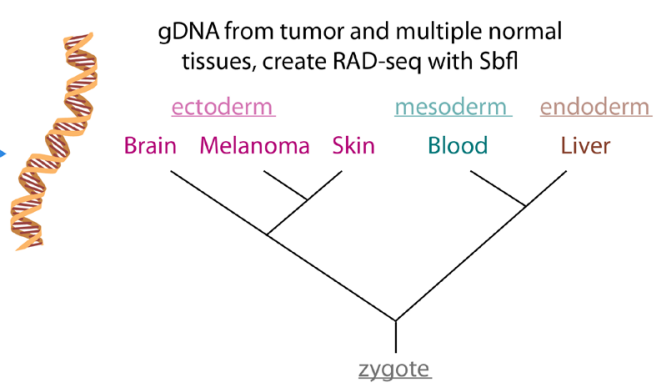

C

\begin{tabular}{llccc}
\hline Genotype & Phenotype & $\begin{array}{c}\text { Restriction } \\
\text { enzyme }\end{array}$ & Tissue & \# mutations \\
\hline Tuebingen & Wild-type & Sbfl & Brain & 0 \\
\hline Tuebingen & Wild-type & Sbfl & Skin & 0 \\
\hline Tuebingen & Wild-type & Sbfl & Blood & 0 \\
\hline Tuebingen & Wild-type & Sbfl & Liver & 0 \\
\hline
\end{tabular}

$\mathrm{E}$

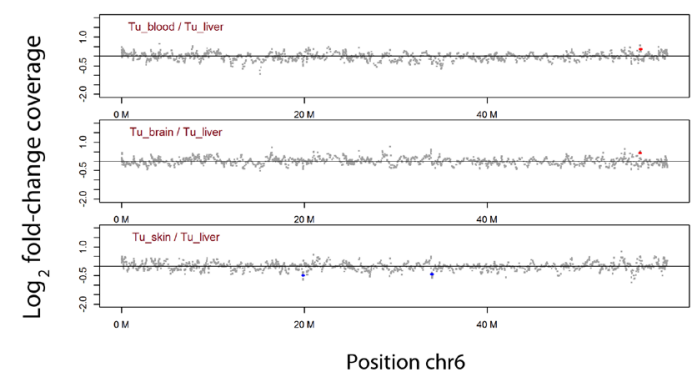

Figure 3: RADseq can identify somatic mosaicism. A. Multiple normal tissues were extracted from both WT and BRAF;p53 fish, including brain, skin, blood, liver, and spleen. Genomic DNA was isolated and RADseq performed using NsiI. As shown in A., these tissues represent ontologically distinct origins from the zygote. B. Mutations were detected in the melanoma as expected. In addition, a somatic mutation was identified and validated in the seemingly-normal brain of the BRAf;p53 fish, an area devoid of BRAF expression. C. No somatic mutations were detected in any of the tissues derived from the WT animal. D., E. The RADseq library was used to detect copy number changes in the above tissues. This revealed two significant copy number amplifications on chromosome 6 in the melanoma, whereas all other tissues from both WT and BRAF;p53 were largely diploid. 

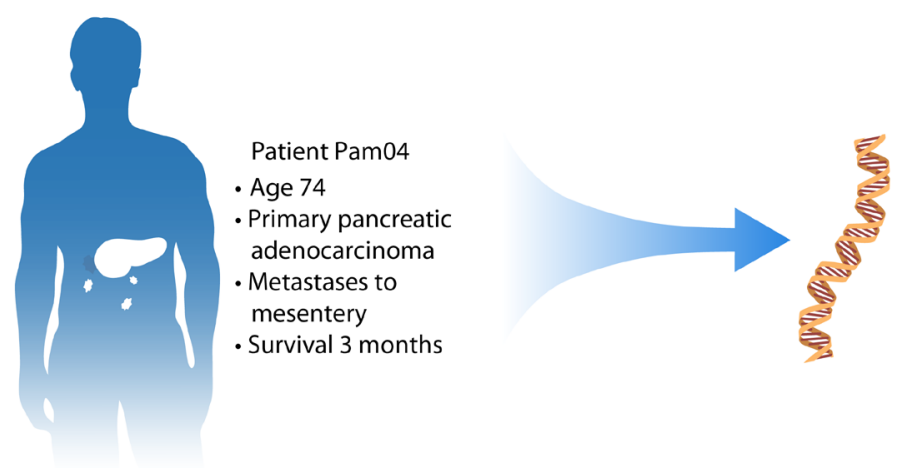

- Extract gDNA from primary metastatic

tumors and 3 normal tissues

- RADseq with Nsil

B

\begin{tabular}{cccccc}
\hline Sample ID & $\begin{array}{c}\text { Restriction } \\
\text { enzyme }\end{array}$ & $\begin{array}{c}\text { Percent genome } \\
\text { represented }\end{array}$ & $\begin{array}{c}\text { \# predicted sites in genome } \\
\text { (\% on target in seq) }\end{array}$ & $\begin{array}{c}\text { Sequencing } \\
\text { depth }\end{array}$ & \# mutations \\
\hline $\begin{array}{c}\text { Primary pancreatic } \\
\text { tumor }\end{array}$ & Nsil & $6 \%$ & $\begin{array}{c}922,636 \\
(96 \% \text { on target })\end{array}$ & $51 \mathrm{x}$ & 164 \\
\hline $\begin{array}{c}\text { Mesenteric } \\
\text { metastasis }\end{array}$ & Nsil & $6 \%$ & $\begin{array}{c}922,636 \\
(97 \% \text { on target })\end{array}$ & $46 \mathrm{x}$ & 168 \\
\hline Normal lung & Nsil & $6 \%$ & $\begin{array}{c}922,636 \\
(97 \% \text { on target })\end{array}$ & $53 x$ & -- \\
\hline Normal skin & Nsil & $6 \%$ & $\begin{array}{c}922,636 \\
(97 \% \text { on target })\end{array}$ & $83 x$ & -- \\
\hline Normal spleen & Nsil & $6 \%$ & 922,636 & $28 \mathrm{x}$ & \\
\hline
\end{tabular}

C

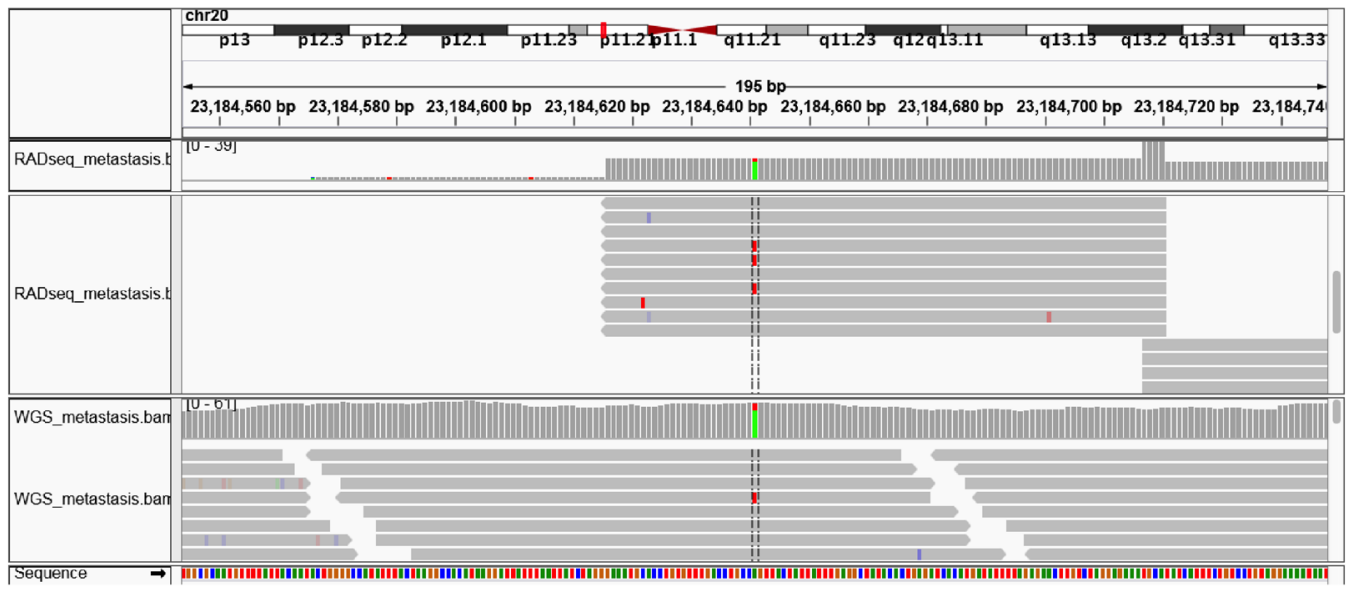

D

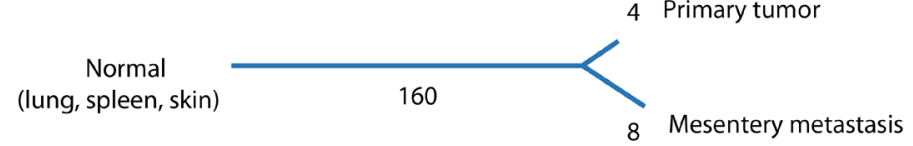

Figure 4: RADseq is adaptable to human cancer specimens. A. Through a rapid autopsy program, tissues were obtained from a patient with metastatic pancreatic ductal adenocarcinoma (PDAC). These included three tumor-free normal tissues (lung, spleen, kidney), the primary pancreatic tumor, and a mesenteric metastasis. Genomic DNA was isolated from these tissues and subject to RADseq using NsiI. B. We identified 168 mutations in the metastasis and 164 mutations in the primary tumor. C. An example IGV plot showing the same mutation on chromosome 20 identified by RADseq (top) and whole-genome sequencing (bottom). RADseq shows bidirectional reads emanating from the cut site, in contrast to the random tiling of reads in the whole-genome approach. D. Mutations in the primary tumor and metastasis were used to construct a phylogenic tree depicting the evolutionary history among the sequenced populations. 160 mutations are present in the primary tumor and metastasis, indicating that these mutations were already present in the ancestral population that gave rise to the two tumors. Four mutations are unique to the primary tumor, and eight mutations are unique to the metastasis, indicating that these mutations occurred after the two populations diverged. 
fish ubiquitously express GFP until exposed to Cre recombinase, which induces a deletion of the GFP coding region and expression of mCherry. We crossed these zebrafish with the (p53 $3^{--;}$mitfa-BRAFV600E ${ }^{+/+}$) zebrafish line that spontaneously develops melanoma. Into this background, we injected an inducible Cre transgene (mitfa:Cre $e^{E R t 2}-S V 40$ ) under the control of the mitfa melanocyte-specific promoter [41]. Exposure to the drug Tamoxifen induces Cre-mediated excision of the GFPlox complex in melanocytes specifically, causing those cells to express mCherry, while the rest of the somatic cells continue to express GFP. We screened for fish that successfully integrated the transgene into their germline and whose offspring show a strong and specific switching of GFP to mCherry expression in melanocytes. The result is a stable line of melanoma-prone zebrafish with inducible fluorophore-based lineage tracing in melanocyte lineages. These fish can be treated with tamoxifen as embryos or adults to induce an irreversible color change in melanocytes. After the drug is removed, the labeled cells continue to express mCherry, and they pass this change on to their daughter cells, resulting in labeled clonal lineages that can be separated from other cells using FACS.

We found that the RADseq method can be used to identify mutations in sorted tumor populations from these animals. We applied tamoxifen to fish with melanoma tumors to label melanocytes in tumors and normal skin before dissection. We dissected tissue, disassociated cells, and used FACS to separate the mCherry+ melanocytes from contaminating stromal cells, and prepared the cells for downstream sequence analysis. We also reserved a portion of each dissected tissue for traditional RADseq analysis of the unsorted population. Nine mutations were identified in both the sorted and unsorted analyses. These mutations show a significantly higher frequency in the sorted $(\mathrm{M}=30.56)$ than the unsorted $(\mathrm{M}=25.67)$ population; $\mathrm{t}(8)=7.72, p<.0001$ (Figure $2 \mathrm{D} / \mathrm{E}$ and Supplemental Table 2) which is consistent with the expectation that the specific sorting of melanocytes allowed us to reduce the number of contaminating normal cells in the sample. We believe this system will provide a powerful system to isolate and study subclonal populations of normal and tumor melanocytes throughout development.

\section{Capturing somatic mosaicism with RADseq}

We dissected and sequenced different tissues from a melanoma-prone zebrafish and a wild-type zebrafish to test whether acquired genetic variation could be also be detected in somatic lineages other than the tumor population, given that the fish were p53 deficient in all tissues. To do so, we dissected two additional normal tissues, brain and liver, to supplement the skin and melanoma samples from one of the transgenic fish described above. We also isolated brain, skin, blood and liver samples from a wild-type zebrafish. Our selection of skin, blood, and liver was based on the fact that these tissues are derived from different developmental germ layers (ectoderm, mesoderm, and endoderm, respectively) and therefore form distinct lineages early in embryonic development and have the most potential to acquire distinct somatic changes (Figure 3A). The brain is derived from the ectoderm along with skin, and we included this additional tissue because acquired somatic mosaicism has been previously described in this organ [42-45]. We used SbfI to RAD-sequence each of these tissues (see Supplemental Table 3 for RADseq metrics), and detected a somatic mutation specific to the brain sample of the transgenic fish (i.e. a polymorphism present in the brain tissue, but not in the liver, skin, or melanoma tumor from that same animal) (Figure 3B). We confirmed the presence of this mutation in the brain (and absence in other tissues) through targeted PCR of the original genomic DNA and additional deep sequencing of the PCR amplicons to $\sim 1000 x$ coverage in a subsequent HiSeq run. The mutation has a frequency of over $20 \%$ in the brain, which indicates that the mutation likely occurred early in development. We did not detect derived mutations in any tissues of the wildtype fish (Figure 3C).

\section{Assessing copy number alterations with RADseq}

RADseq has been previously shown to be an effective tool to identify copy number alterations in human tumors [46]. To our knowledge, this study by Zheng et al. is the only previous application of the RADseq method to cancer. We analyzed the SbfI-digested RADseq samples from normal and melanoma tissue from melanoma-prone and wild-type fish for evidence of copy number alterations. We identified two regions of chromosome six in the melanoma tumor sample that show strong evidence of amplification (Figure 3D). These regions contain 116 genes, and are not among previouslydocumented recurrently amplified regions in engineered zebrafish melanomas [35]. Although we cannot evaluate the functional importance of copy number alterations from sequence data alone, the amplified regions do contain several genes of interest including the zebrafish ortholog of the human transcription factor, Myc, which is a frequently mutated oncogene in humans including amplification/overexpression in $6 \%$ of melanomas in the TCGA database [47, 48].

We did not detect any copy number changes in any of the normal tissues of the melanoma-prone fish or the wild-type fish (Figure 3E). RADseq has specific advantages for studies of copy number alterations because the selection of restriction enzyme sets the resolution at which copy number change events can be detected. A frequently-cutting enzyme will produce a high density of markers throughout the genome to identify amplifications and deletions of small genomic regions. A rare cutter will produce a lower density of markers spread across the genome, and can be used to identify amplifications 
and deletions of large genomic regions, with very low sequencing cost.

\section{Human pancreatic cancer samples from primary and metastatic sites}

A unique feature of RADseq is that the same protocol and reagents can be used for any species because there are no sequence-specific capture steps (e.g. exonic baits) involved. We used the enzyme Nsil to generate RADseq libraries for tissue samples obtained from a human pancreatic cancer patient through a rapid autopsy program (Figure 4A) [11]. In silico analysis using the human reference genome hg19 predicts 922,636 NsiI cut sites in the genome. Sequencing 100bp flanking both directions of each cut site results in representation of approximately $6 \%$ of the human genome. Note that this is a larger fraction of genome coverage than is targeted by common exome capture kits which typically represent approximately $50-65 \mathrm{Mb}(\sim 2 \%$ of the human genome). Similar to performance in zebrafish described above, RADseq showed very high efficiency with the NsiI enzyme in human tissues (see Supplemental Table 4 for RADseq metrics). After filtering for quality, 92.4\% of reads successfully mapped to the human reference genome. $97.0 \%$ of these reads mapped to the predicted cut site regions, and $99.28 \%$ of predicted cut site regions received sequence coverage. The average depth of coverage was $\sim 50 \mathrm{x}$ ( $52 \mathrm{x}$ mean, $49.6 \mathrm{x}$ median).

The tissues included in this study were primary pancreatic tumor, mesentery metastasis, and normal spleen. These tissues had already undergone wholegenome sequencing (WGS), and thus provided an independent and comprehensive sequence dataset that we could use to validate RADseq performance for identifying mutations. After filtering for quality, we aligned reads to the reference genome using BWA, and we called mutations with MuTect. We identified 168 mutations distinguishing the metastasis from the normal sample and 164 mutations distinguishing the primary tumor from the normal sample (Figure 4B and Supplemental Table 5 for a list of all mutations detected). 116 (67\%) of the 172 total unique mutations that we detected fall in intergenic regions, $53(31 \%)$ are in introns, and $3(2 \%)$ are in exons (an example of which is shown in Figure 4C). This distribution is consistent with the composition of the human genome, and supports the prediction that RADseq loci are distributed approximately randomly in the genome and that the majority of mutations detected will be functionally neutral. Of the three exonic mutations, one was silent and two were missense mutations, in codon 12 of KRAS and exon 49 of FBN1. The mutation that we detected in codon 12 of KRAS (G12D) is a common and important driver in pancreatic and other cancers and is concordant with that found by whole genome sequencing of these same samples [49-51]. Although RADseq is not an efficient method to search for driver genes in cancer, there happens by chance to be an NsiI cut site adjacent to this important region, thus cut sites adjacent to hot spots can also identify somatic variants of importance.

To compare results between RADseq and WGS, we independently analyzed sequence data from the wholegenome sequence library. All reads were aligned against the human reference genome, and we used MuTect to identify single nucleotide variants. For efficiency, we restricted mutation calling to regions of the genome adjacent to NsiI cut sites. The whole-genome sequence libraries confirmed the presence of $100 \%$ of the RADseqdetected variants.

We used mutation information from primary tumor and metastasis to infer a phylogenetic tree that depicts the evolutionary history underlying tumor progression (Figure 4D). 160 mutations occur before the primary tumor and metastasis lineages diverge. 8 mutations occur along the metastasis lineage, and 4 mutations occur in the primary tumor lineage after its split from the metastasis lineage. These results are consistent with previous studies of pancreatic cancer patients that show a long history of shared mutations among primary tumors and metastases, with fewer unique mutations distinguishing individual tumors within patients [11].

\section{DISCUSSION}

When applied to the appropriate research questions, RADSeq provides an efficient, flexible and cost effective method to utilize the power of next-generation sequencing technologies to gain new insights into the ecological and evolutionary dynamics of cancer. Some additional details regarding study design and analysis will be useful for cancer biologists employing this technique.

Study design: When designing a RADseq experiment, the choice of restriction enzyme will determine the number of loci represented in each sequence library. It is important to have an expectation for the number of cut sites produced by potential restriction enzymes in order to design an experiment that will provide sufficient genetic resolution for the research question, and to anticipate how much sequencing will be required to achieve a given depth of coverage per site. The simplest estimate is that an 8 -cutter will cut every $4^{8}=65,536 \mathrm{bp}$ and a 6 -cutter will cut every $4^{6}=4,096 \mathrm{bp}$, but many genomes and enzymes will deviate significantly from this expectation [19]. The calculation can be improved by accounting for the GC content of the cut site and genome under study, however, the most accurate estimates for numbers of cut sites can be obtained through in silico analysis from a published reference genome for the species under study. Computational tools exist to help plan an appropriate sequencing effort for a given target depth by estimating the number of loci expected for a 
given protocol and genome [52]. The number of bases represented per genome will also be determined by the read length of the sequencing technology (typically up to $150 \mathrm{bp}$ reads currently with Illumina, for example). Most questions can be sufficiently addressed with short reads and single-end sequencing, but longer reads can also be obtained by assembling contigs from paired-end sequence reads.

Quality and quantity of starting material: RADseq library preparation protocols have been optimized for high-molecular weight genomic DNA, and are not expected to perform as well with highly degraded DNA such as that obtained from FFPE tissue [53]. In degraded samples, small fragments of starting DNA not adjacent to cut sites may end up in the sequencing library and waste sequencing effort on off-target loci. Also, the mechanical shearing step to produce fragments of optimal size for the sequencing platform works best with relatively large fragments present after enzyme digestion. In addition to high quality DNA, a large quantity of starting DNA is beneficial because it can reduce the number of PCR cycles required for the final step of library prep to reduce PCR bias/duplicates. Early RADseq papers recommend as much as lug of starting DNA, but more recent studies have shown successful library prep with as little as 50 100ng DNA per sample [19].

Error: RADseq is subject to many of the same sources of error that challenge all high-throughput sequencing studies of cancer genomics. Type II error or 'false negative' mutation calls can occur if a mutation is present at low frequency within the tumor population and sequencing depth is insufficient to resolve the variant. RADseq may be less susceptible to this type of false negative than whole exome sequencing or whole genome sequencing because the cost-effectiveness of RADSeq enables greater sequencing depth per site. False negative mutation calls can also occur when tumors contain genetic heterogeneity that is not captured in a single biopsy. RADseq again may have advantages over WES and WGS with regards to this problem because the more cost-effective approach enables the sequencing of multiple spatially-independent tumor samples (multiregion sequencing) instead of a single biopsy. Type I error or 'false positive' mutation calls also need to be considered in all high-throughput sequencing studies of tumors. False positives due to mapping errors of reads to the reference genome can be minimized in RADseq with the same bioinformatics preprocessing tools designed to address these problems in whole genome and whole exome studies, such as GATK indel realignment [54]. We find that mapping errors are easier to identify with RADseq than other sequencing methods because the palindromic nature of the restriction enzyme cut sites should result in two sets of reads independently mapping to the same genomic region - one from each direction of the cut site. False positive mutation calls can also result from PCR errors occurring during the library preparation. These errors need to be addressed differently in RADseq than whole genome or whole exome studies, as we discuss in detail below.

PCR errors and duplicates: Like most nextgeneration sequencing library preparation protocols, RADseq methods include a PCR step to enrich for fragments that have successfully ligated sequencing adapters. This amplification can potentially lead to erroneous downstream mutation calls, if duplicates that contain PCR errors appear as mutations. The standard method to eliminate PCR duplicates from whole-genome and whole-exome sequence libraries involves removing reads that start and end at the exact same genomic position. This method is not appropriate for RADseq libraries because reads consistently begin at the restriction enzyme cut site. To eliminate PCR duplicates from RADseq data, several alternative methods are available. One option is to use paired-end sequencing (i.e. sequence the randomlysheared end of the DNA as well as the cut-site end, to remove reads with identical start and end positions). Alternatively, parallel PCR reactions can be performed for each sample and sequenced in separate lanes. Our preferred method is to use multiple barcodes for each individual sample (introduced as a mixture during library prep) and ensure that mutations identified at a particular locus are confirmed by reads containing different barcodes (and thus not the result of PCR error and duplication). The use of multiple barcodes also increases the complexity of the library, which leads to better cluster identification on the Illumina platform. A final way to eliminate PCR duplicates is to eliminate the PCR step of library prep altogether, but this requires higher starting quantities of genomic DNA.

Reagents: The RADseq protocol requires an initial financial investment in specialized barcoded adapters, but adapter sequences are non-proprietary and a single set of oligonucleotides is sufficient for a large number of libraries. Additionally, the same set of adapters can be used for compatible sets of enzymes that leave the same sticky end. For example, the two enzymes used in this study (SbfI and NsiI) leave the same ACGT overhang, so we were able to use a single set of adapter oligos to produce libraries from both restriction enzymes. The same set of barcoded adapter oligos can also be used for different species, as we demonstrate with human and zebrafish.

\section{CONCLUSIONS}

Many studies of evolution in cancer are more limited by the number of individuals or tissues sampled than by the density of markers in the genome, and for these studies RADseq will be an especially useful tool. Like other reduced-representation approaches, RADseq provides advantages over whole genome sequencing, such as greater depth of coverage per locus and the sequencing 
of higher numbers of samples for a given budget. RADseq also offers an alternative to whole-exome sequencing because it captures more neutrally-evolving sites and thus produces more reliable markers to measure intrinsic mutation rates and to infer phylogenetic relationships than sites under strong positive or purifying selection. Our results demonstrate that RADseq can be an effective tool to identify single-nucleotide variants and copy number alterations in humans and animal models. Our detection of somatic changes in normal tissue of adult zebrafish also indicates that RADseq can be useful for studies of somatic mosaicism in development. The transgenic zebrafish that we created can also be used with RADseq to detect mutations in subclonal lineages.

\section{EXPERIMENTAL PROCEDURES}

\section{Animal husbandry}

All zebrafish were housed in a temperature (28.5C) and light-controlled (14h on, 10h off) room. Fish were housed at a density of 5 fish per liter, and fed 3 times per day using brine shrimp and pelleted zebrafish food. All procedures adhered to IACUC protocol \#12-05-008 through Memorial Sloan Kettering Cancer Center, as described previously [55].

\section{Zebrafish tissue dissection and DNA extraction}

Adult zebrafish were anesthetized in $0.2 \%$ Tricaine and then euthanized by incubation in ice water for 15 minutes. Tissues were dissected according to the protocol and video published at http://www.jove.com/video/1717/ [56]. Blood was collected according to the protocol and video published at http://www.jove.com/video/3865/ [57]. DNA was extracted from dissected tumors and normal tissue using the DNeasy Blood \& Tissue Kit (Qiagen), with an RNAse A treatment step.

\section{Library preparation}

RADseq libraries were prepared from extracted DNA according to the method described by Etter et al [22, 23] using the enzymes SbfI-HF and NsiI (New England Biolabs), with $1 \mu \mathrm{g}$ of DNA starting material. The shearing step was performed on a Bioruptor+ sonication device (Diagenode) with 10 cycles of 30 seconds on, 1 minute off (high setting). Zymo DNA Clean and Concentrator kits (Zymo Research) were used for each clean-up step. The final PCR amplifications were run for 12 cycles. We did not pool samples after the P1 ligation - all samples were barcoded and processed separately until final quantification with a Qubit fluorometer (Thermo Scientific) before being combined proportionately for multiplexed sequencing. Four different barcodes were used for each sample to increase library complexity and assist in eliminating PCR errors in downstream processing.

\section{Human tissue dissection and library prep}

Tissue sample processing. The patient and tissues were collected through the Johns Hopkins Gastrointestinal Cancer Rapid Medical Donation program [58]. Informed consent was obtained. Upon opening the body cavity, the entire primary tumor and remaining normal pancreas were dissected along with each metastasis. All tumor and normal tissues were flash-frozen in liquid nitrogen and stored at $-80 \mathrm{oC}$. The primary tumor was sliced $(0.5 \mathrm{~cm}$ thick) and sectioned into $1 \mathrm{x} 1 \mathrm{~cm}$ samples as described previously [11]. Macrodissection of each metastasis removed non-neoplastic tissue.

Genomic DNA extraction and quantification. A phenol and chloroform method was used to extract genomic DNA (gDNA) from each tumor sample followed by LINE assay quantification (i.e. counting long interspersed elements (LINE) using real-time PCR). The LINE forward primer used was 5'-AAAGCCGCTCAACTACATGG-3' and the reverse primer was 5'-TGCTTTGAATGCGTCCCAGAG-3'. The PCR protocol implemented was $50^{\circ} \mathrm{C}$ for $2 \mathrm{~min}, 95^{\circ} \mathrm{C}$ for $2 \mathrm{~min}, 40$ cycles of $94^{\circ} \mathrm{C}$ for $10 \mathrm{~s}, 58^{\circ} \mathrm{C}$ for $15 \mathrm{~s}$, and $70^{\circ} \mathrm{C}$ for $30 \mathrm{~s}, 95^{\circ} \mathrm{C}$ for $15 \mathrm{~s}$, and $60^{\circ} \mathrm{C}$ for $30 \mathrm{~s}$. All PCR reactions used Platinum SYBR Green qPCR mastermix (Invitrogen).

Whole genome sequencing and alignment. Sequencing libraries were prepared using standard methods for each gDNA sample. Whole genome sequencing (WGS) utilized an Illumina Hi-Seq 2000 platform for 60x target coverage. Sequencing reads were aligned to the hg19 human reference genome.

\section{Transgenic zebrafish generation}

Melanoma-prone zebrafish $\operatorname{Tg}$ (mitfa:BRAF(V600E)); $p 53^{-/}$[28] were crossed with the ubi;Switch zebrafish line (ubi:loxP-GFP-loxP-mCherry) [39]. A plasmid containing mitf: $\mathrm{Cr} e^{E R t 2} ; S V 40$ was created in the pDestTol2CG2 destination vector with the Gateway system [59]. This plasmid $(25 \mathrm{ng} / \mu \mathrm{l})$ was injected at the single cell stage using a micropipette along with Tol2 RNA (20ng/ $\mu$ l) according to previously described protocols $[39,60]$. Embryos were treated with $20 \mu \mathrm{m}$ 4-OHT (Sigma) at $50 \%$ epiboly. At 24 hours, the drug was refreshed and $0.003 \%$ of 1-phenyl-2-thiourea (PTU) was applied to temporarily block pigment production in melanocytes and aid in the visualization of fluorophores [61]. Larvae were imaged at $3 \mathrm{dpf}$ to look for color change in melanocytes from GFP to mCherry. Fish that showed 
strong and specific switching were grown up and then incrossed to produce an F2 population that was also treated and imaged to confirm incorporation of the transgene in the germline. These fish were grown up and genotyped to select for individuals that carry both homozygous mutations required for the development of melanoma as well as demonstrating the strong and specific switching of melanocytes from GFP to mCherry after tamoxifen treatment. These fish were used to generate a stable line and for the sequencing in this study.

\section{Tissue digestion and FACS sorting}

Adult fish were dissected as described above and the excised tissue was placed in an Eppendorf tube containing $500 \mu \mathrm{L}$ liberase diluted in DMEM media without serum (final: $0.15 \mathrm{U} / \mathrm{ml}$ ). A mini pestle was used to mechanically dissociate the cells. Tubes were then incubated at $37^{\circ} \mathrm{C}$ until the cells appeared to be completely dissociated in the solution. $500 \mu \mathrm{L}$ of DMEM10 media (with serum) was added to stop the liberase activity. The cells were then pelleted by centrifugation at $500 \mathrm{RCF}$ for $5 \mathrm{~min}$ in a refrigerated centrifuge. The supernatant was discarded and cells resuspended in PBS buffer. The solution was filtered into a FACS tube through a $40 \mu \mathrm{m}$ mesh filter, with Dapi (1:100). mCherry positive cells were separated from GFP positive cells on an Aria III FACS machine with gating set by controls from pure populations of zebrafish in vitro cell lines. DNA was extracted from sorted cells as described above.

\section{Sequencing method}

All sequencing was performed on an Illumina HiSeq 2500 at the University of Oregon. Single-end reads of $100 \mathrm{bp}$ or $150 \mathrm{bp}$ were generated, and raw reads were exported for downstream analysis.

\section{Informatics analysis}

Filtering for quality. Each lane of data was processed with STACKS $[62,63]$, through the process radtags pipeline, which checks for intact barcodes and cut-sites and sorts reads by barcodes into separate files. The pipeline also filters for quality using a sliding window approach and reads were discarded if the score dropped below $90 \%$ probability of being correct. The sliding window was set to $15 \%$ of the length of the read. Preprocessing. Reads passing the initial quality filter were pre-processed as described in GATK "best practices" $[54,64,65]$ with the exception of the de-duplication step because that step is incompatible with RADseq data (see removing PCR duplicates above). Reads were aligned to the zebrafish (danRer7) or human (hg19) reference genomes using BWA [33]. Mutation calling. Mutationcalling was done using MuTect [34]. Manual curation. The mutation lists generated by MuTect were curated via manual inspection of the alignment files at each location using the Integrative Genomics Viewer (IGV) [66]. Mutations in regions with problematic mapping or asymmetrical coverage of RADseq reads on each side of the cut site were omitted.

\section{Copy-number alteration}

Restriction sites were located in the zebrafish genome (danRer7 assembly) via in silico digestion of SbfI. Only reads that perfectly matched the location of restriction sites were retained to calculate the coverage of the sites. To depress the fluctuation of read depth, we merged 5 consecutive restriction sites as a unit, and walked along the chromosome via a sliding-window to calculate average count read number in each unit. The read depth ratio for each unit of the tested sample was calculated by dividing corresponding value in liver sample of melanoma-prone strain or wild-type strain after normalization of total reads counts. The $\mathrm{R}$ package "DNAcopy" was used to segment the chromosome via read depth ratio of each tested sample. To resolve the hyper-segmentation, the FastCall algorithm [67] was used to merge neighboring segments with similar copy numbers.

\section{Authors' contributions}

RMW and EBP conceived the study. EBP carried out all zebrafish experiments, RAD-sequencing and analysis, and wrote the manuscript. AMM and CID provided the pancreatic tumor samples and whole-genome sequencing data for those samples. CKK created and provided the mitfa-CreERT2 plasmid. JC and CZ performed the copy number analysis on the zebrafish tumors.

\section{ACKNOWLEDGMENTS}

The authors would like to thank Wenjing $\mathrm{Wu}$ for assistance in preparing figures for publication. We would also like to thank Paul Etter for guidance on RADseq library prep and William Lee for help with sequence analysis. We are grateful to Doug Turnbull and the genomics core facility at the University of Oregon for their expertise in RAD-sequencing.

\section{ETHICS APPROVAL AND CONSENT TO PARTICIPATE}

All animal studies were approved by the Memorial Sloan Kettering IACUC under protocol number 12- 
05-008. All procedures had ethics approval from the institutional board.

\section{Availability of data and material}

All sequence data will be deposited in the NCBI Sequence Read Archive.

\section{CONFLICTS OF INTEREST}

The authors declare no potential conflicts of interest

\section{FUNDING}

This work was supported by the NIH Director's New Innovator Award (DP2CA186572), the Mentored Clinical Scientist Research Career Development Award (K08AR055368), the Melanoma Research Alliance Young Investigator Award, an AACR/ASCO Young Investigator Award, and the Alan and Sandra Gerry Metastasis Research Initiative at the Memorial Sloan Kettering Cancer Center. EBP was supported by the Translational Research in Oncology Training Award (5T32CA160001) and the NIH Loan Repayment Program. AMM was supported by F31CA180682 and the First Eagle Foundation. These funding agencies were not involved in the design or interpretation of the data.

\section{Statement of significance}

Cancer evolution plays a significant role in drug resistance and metastasis, but its study is limited by current approaches. We implement RADseq to analyze cancer evolution, which allows for an unbiased view of genome evolution in time and space. It can be applied to human and animal models, opening up the field to new investigators.

\section{REFERENCES}

1. Nowell PC. The clonal evolution of tumor cell populations. Science. 1976; 194: 23-28.

2. Greaves M. Evolutionary Determinants of Cancer. Cancer Discovery. 2015; 5: 806-820.

3. Aparicio S, Caldas C. The Implications of clonal genome evolution for cancer medicine. New England Journal of Medicine. 2013; 368: 842-851.

4. Bernards R, Weinberg RA. A progression puzzle. Nature. 2002; 418: 823

5. Frank SA, Nowak MA. Problems of somatic mutation and cancer. Bioessays. 2004; 26: 291-299.

6. Greaves M, Maley CC. Clonal evolution in cancer. Nature. 2012; 481: 306-813.
7. Tomasetti C, Vogelstein B, Parmigiani G. Half or more of the somatic mutations in cancers of self-renewing tissues originate prior to tumor initiation. Proceedings of the National Academy of Sciences of the United States of America. 2013; 110: 1999-2004.

8. Bozic I, Gerold JM, Nowak MA. Quantifying clonal and subclonal passenger mutations in cancer evolution. PLoS Comput Biol. 2016; 12.

9. Waclaw B, Bozic I, Pittman ME, Hruban RH, Vogelstein B, Nowak MA. A spatial model predicts that dispersal and cell turnover limit intratumour heterogeneity. Nature. 2015; 525: 261-264.

10. Marusyk A, Tabassum DP, Altrock PM, Almendro V, Michor F, Polyak K. Non-cell-autonomous driving of tumour growth supports sub-clonal heterogeneity. Nature. 2014; 514: 54-58.

11. Yachida S, Jones S, Bozic I, Antal T, Leary R, Fu B, Kamiyama M, Hruban RH, Eshleman JR, Nowak MA, Velculescu VE, Kinzler KW, Vogelstein B, et al. Distant metastasis occurs late during the genetic evolution of pancreatic cancer. Nature. 2010; 467: 1114-1117.

12. Gerlinger M, Rowan AJ, Horswell S, Larkin J, Endesfelder D, Gronroos E, Martinez P, Matthews N, Stewart A, Tarpey P, Varela I, Phillimore B, Begum S, et al. Intratumor heterogeneity and branched evolution revealed by multiregion sequencing. N Engl J Med. 2012; 366: 883-892.

13. Swanton C. Cancer Evolution constrained by mutation order. New England Journal of Medicine. 2015; 372: 661663.

14. Zhao ZM, Zhao B, Bai Y, Iamarino A, Gaffney SG, Schlessinger J, Lifton RP, Rimm DL, Townsend JP. Early and multiple origins of metastatic lineages within primary tumors. Proc Natl Acad Sci U S A. 2016; 113: 2140-2145.

15. Badri H, Pitter K, Holland EC, Michor F, Leder K. Optimization of radiation dosing schedules for proneural glioblastoma. J Math Biol. 2016; 72: 1301-1336.

16. Michor F, Beal K. Improving cancer treatment via mathematical modeling: Surmounting the challenges Is worth the effort. Cell. 2015; 163: 1059-1063.

17. Enriquez-Navas PM, Kam Y, Das T, Hassan S, Silva A, Foroutan P, Ruiz E, Martinez G, Minton S, Gillies RJ, Gatenby RA. Exploiting evolutionary principles to prolong tumor control in preclinical models of breast cancer. Sci Transl Med. 2016; 8: 327.

18. Ali OA, O'Rourke SM, Amish SJ, Meek MH, Luikart G, Jeffres C, Miller MR. RAD Capture (Rapture): Flexible and efficient sequence-based genotyping. Genetics. 2016; 202: $389-400$

19. Andrews KR, Good JM, Miller MR, Luikart G, Hohenlohe PA. Harnessing the power of RADseq for ecological and evolutionary genomics. Nat Rev Genet. 2016; 17: 81-92.

20. Baird NA, Etter PD, Atwood TS, Currey MC, Shiver AL, Lewis ZA, Selker EU, Cresko WA, Johnson EA. Rapid SNP discovery and genetic mapping using sequenced RAD 
markers. PLoS One. 2008; 3: e3376.

21. Davey JW, Hohenlohe PA, Etter PD, Boone JQ, Catchen JM, Blaxter ML. Genome-wide genetic marker discovery and genotyping using next-generation sequencing. Nat Rev Genet. 2011; 12: 499-510.

22. Etter PD, Bassham S, Hohenlohe PA, Johnson EA, Cresko WA. SNP discovery and genotyping for evolutionary genetics using RAD sequencing. Methods Mol Biol. 2011; 772: 157-78.

23. Etter PD, Preston JL, Bassham S, Cresko WA, Johnson EA. Local de novo assembly of RAD paired-end contigs using short sequencing reads. PLoS One. 2011; 6: e18561.

24. Peterson BK, Weber JN, Kay EH, Fisher HS, Hoekstra HE. Double digest RADseq: an inexpensive method for de novo SNP discovery and genotyping in model and non-model species. PLoS One. 2012; 7: e37135.

25. Felsenstein J. Inferring phylogenies. Sunderland, Mass.: Sinauer Associates; 2014.

26. Brannon AR, Vakiani E, Sylvester BE, Scott SN, McDermott G, Shah RH, Kania K, Viale A, Oschwald DM, Vacic V, Emde AK, Cercek A, Yaeger R, et al. Comparative sequencing analysis reveals high genomic concordance between matched primary and metastatic colorectal cancer lesions. Genome Biol. 2014; 15: 454.

27. Goswami RS, Patel KP, Singh RR, Meric-Bernstam F, Kopetz ES, Subbiah V, Alvarez RH, Davies MA, Jabbar KJ, Roy-Chowdhuri S, Lazar AJ, Medeiros LJ, Broaddus $\mathrm{RR}$, et al. Hotspot mutation panel testing reveals clonal evolution in a study of 265 paired primary and metastatic tumors. Clin Cancer Res. 2015; 21: 2644-2651.

28. White RM, Cech J, Ratanasirintrawoot S, Lin CY, Rahl PB, Burke CJ, Langdon E, Tomlinson ML, Mosher J, Kaufman C, Chen F, Long HK, Kramer M, et al. DHODH modulates transcriptional elongation in the neural crest and melanoma. Nature. 2011; 471: 518-522.

29. Patton EE, Widlund HR, Kutok JL, Kopani KR, Amatruda JF, Murphey RD, Berghmans S, Mayhall EA, Traver D, Fletcher CD, Aster JC, Granter SR, Look AT, et al. BRAF mutations are sufficient to promote nevi formation and cooperate with p53 in the genesis of melanoma. Curr Biol. 2005; 15: 249-254.

30. Howe K, Clark MD, Torroja CF, Torrance J, Berthelot C, Muffato M, Collins JE, Humphray S, McLaren K, Matthews L, McLaren S, Sealy I, Caccamo M, et al. The zebrafish reference genome sequence and its relationship to the human genome. Nature. 2013; 496: 498-503.

31. Guryev V, Koudijs MJ, Berezikov E, Johnson SL, Plasterk RH, van Eeden FJ, Cuppen E. Genetic variation in the zebrafish. Genome Res. 2006; 16: 491-497.

32. Brown KH, Dobrinski KP, Lee AS, Gokcumen O, Mills RE, Shi X, Chong WW, Chen JY, Yoo P, David S, Peterson SM, Raj T, Choy KW, et al. Extensive genetic diversity and substructuring among zebrafish strains revealed through copy number variant analysis. Proc Natl Acad Sci U S A.
2012; 109: 529-534.

33. Li H, Durbin R. Fast and accurate short read alignment with Burrows-Wheeler transform. Bioinformatics. 2009; 25: 1754-1760.

34. Cibulskis K, Lawrence MS, Carter SL, Sivachenko A, Jaffe D, Sougnez C, Gabriel S, Meyerson M, Lander ES, Getz G. Sensitive detection of somatic point mutations in impure and heterogeneous cancer samples. Nature Biotechnology. 2013; 31: 213-219.

35. Yen J, White RM, Wedge DC, Van Loo P, de Ridder J, Capper A, Richardson J, Jones D, Raine K, Watson IR, Wu CJ, Cheng J, Martincorena I, et al. The genetic heterogeneity and mutational burden of engineered melanomas in zebrafish models. Genome Biol. 2013; 14: R113.

36. Almendro V, Marusyk A, Polyak K. Cellular heterogeneity and molecular evolution in cancer. Annu Rev Pathol. 2013; 8: 277-302.

37. Aran D, Sirota M, Butte AJ. Systematic pan-cancer analysis of tumour purity. Nat Commun. 2015; 6: 8971.

38. Gawad C, Koh W, Quake SR. Single-cell genome sequencing: current state of the science. Nat Rev Genet. 2016; 17: 175-188.

39. Mosimann C, Kaufman CK, Li PL, Pugach EK, Tamplin OJ, Zon LI. Ubiquitous transgene expression and Crebased recombination driven by the ubiquitin promoter in zebrafish. Development. 2011; 138: 169-77.

40. Mosimann C, Zon LI. Advanced zebrafish transgenesis with Tol2 and application for Cre/lox recombination experiments. Methods Cell Biol. 2011; 104: 173-194.

41. Lister JA, Robertson CP, Lepage T, Johnson SL, Raible DW. Nacre encodes a zebrafish microphthalmia-related protein that regulates neural-crest-derived pigment cell fate. Development. 1999; 126: 3757-3767.

42. De S. Somatic mosaicism in healthy human tissues. Trends in Genetics. 2011; 27: 217-223.

43. Jacobs KB, Yeager M, Zhou WY, Wacholder S, Wang ZM, Rodriguez-Santiago B, Hutchinson A, Deng X, Liu CW, Horner MJ, Cullen M, Epstein CG, Burdett L, et al. Detectable clonal mosaicism and its relationship to aging and cancer. Nature Genetics. 2012; 44: 651.

44. Lupski JR. Genome mosaicism-one human, multiple genomes. Science. 2013; 341: 358-359.

45. Youssoufian H, Pyeritz RE. Mechanisms and consequences of somatic mosaicism in humans. Nature Reviews Genetics. 2002; 3: 748-758.

46. Zheng CH, Miao XX, Li YN, Huang Y, Ruan J, Ma X, Wang L, Wu CI, Cai J. Determination of genomic copy number alteration emphasizing a restriction site-based strategy of genome re-sequencing. Bioinformatics. 2013; 29: 2813-2821.

47. Cerami E, Gao J, Dogrusoz U, Gross BE, Sumer SO, Aksoy BA, Jacobsen A, Byrne CJ, Heuer ML, Larsson E, Antipin Y, Reva B, Goldberg AP, et al. The cBio cancer genomics 
portal: an open platform for exploring multidimensional cancer genomics data. Cancer Discov. 2012; 2: 401-404.

48. Gao J, Aksoy BA, Dogrusoz U, Dresdner G, Gross B, Sumer SO, Sun Y, Jacobsen A, Sinha R, Larsson E, Cerami E, Sander C, Schultz N. Integrative analysis of complex cancer genomics and clinical profiles using the cBioPortal. Sci Signal. 2013; 6: pl1.

49. Stolze B, Reinhart S, Bulllinger L, Frohling S, Scholl C. Comparative analysis of KRAS codon 12, 13, 18, 61, and 117 mutations using human MCF10A isogenic cell lines. Sci Rep. 2015; 5: 8535.

50. Margonis GA, Kim Y, Spolverato G, Ejaz A, Gupta R, Cosgrove D, Anders R, Karagkounis G, Choti MA, Pawlik TM. Association Between Specific mutations in KRAS codon 12 and colorectal liver metastasis. JAMA Surg. 2015; 150: 722-729.

51. Smit VT, Boot AJ, Smits AM, Fleuren GJ, Cornelisse CJ, Bos JL. KRAS codon 12 mutations occur very frequently in pancreatic adenocarcinomas. Nucleic Acids Res. 1988; 16: 7773-7782.

52. Lepais O, Weir JT. SimRAD: an R package for simulationbased prediction of the number of loci expected in RADseq and similar genotyping by sequencing approaches. Mol Ecol Resour. 2014; 14: 1314-1321.

53. Graham CF, Glenn TC, McArthur AG, Boreham DR, Kieran T, Lance S, Manzon RG, Martino JA, Pierson T, Rogers SM, Wilson JY, Somers CM. Impacts of degraded DNA on restriction enzyme associated DNA sequencing (RADSeq). Mol Ecol Resour. 2015; 15: 1304-1315.

54. McKenna A, Hanna M, Banks E, Sivachenko A, Cibulskis K, Kernytsky A, Garimella K, Altshuler D, Gabriel S, Daly M, DePristo MA. The Genome Analysis Toolkit: a MapReduce framework for analyzing next-generation DNA sequencing data. Genome Res. 2010; 20: 1297-1303.

55. Heilmann S, Ratnakumar K, Langdon EM, Kansler ER, Kim IS, Campbell NR, Perry EB, McMahon AJ, Kaufman CK, van Rooijen E, Lee W, Iacobuzio-Donahue CA, Hynes $\mathrm{RO}$, et al. A quantitative system for studying metastasis using transparent zebrafish. Cancer Res. 2015; 75: 42724282 .

56. Gupta T, Mullins MC. Dissection of organs from the adult zebrafish. J Vis Exp. 2010; 37.

57. Pedroso GL, Hammes TO, Escobar TD, Fracasso LB, Forgiarini LF, da Silveira TR. Blood collection for biochemical analysis in adult zebrafish. J Vis Exp. 2012: e3865.
58. Embuscado EE, Laheru D, Ricci F, Yun KJ, de Boom Witzel S, Seigel A, Flickinger K, Hidalgo M, Bova GS, Iacobuzio-Donahue CA. Immortalizing the complexity of cancer metastasis: genetic features of lethal metastatic pancreatic cancer obtained from rapid autopsy. Cancer Biol Ther. 2005; 4: 548-554.

59. Katzen F. Gateway $((\mathrm{R}))$ recombinational cloning: a biological operating system. Expert Opin Drug Discov. 2007; 2: 571-589.

60. Suster ML, Kikuta H, Urasaki A, Asakawa K, Kawakami K. Transgenesis in zebrafish with the tol 2 transposon system. Methods Mol Biol. 2009; 561: 41-63.

61. Westerfield M. The zebrafish book : a guide for the laboratory use of zebrafish (Brachydanio rerio). Eugene, OR: M. Westerfield; 1993.

62. Catchen J, Hohenlohe PA, Bassham S, Amores A, Cresko WA. Stacks: an analysis tool set for population genomics. Mol Ecol. 2013; 22: 3124-3140.

63. Catchen JM, Amores A, Hohenlohe P, Cresko W, Postlethwait JH. Stacks: building and genotyping loci de novo from short-read sequences. G3. 2011; 1: 171-182.

64. DePristo MA, Banks E, Poplin R, Garimella KV, Maguire JR, Hartl C, Philippakis AA, del Angel G, Rivas MA, Hanna M, McKenna A, Fennell TJ, Kernytsky AM, et al. A framework for variation discovery and genotyping using next-generation DNA sequencing data. Nat Genet. 2011; 43: 491-498.

65. Van der Auwera GA, Carneiro MO, Hartl C, Poplin R, Del Angel G, Levy-Moonshine A, Jordan T, Shakir K, Roazen D, Thibault J, Banks E, Garimella KV, Altshuler D, et al. From FastQ data to high confidence variant calls: the Genome Analysis Toolkit best practices pipeline. Curr Protoc Bioinformatics. 2013; 11: 1- 33.

66. Thorvaldsdottir H, Robinson JT, Mesirov JP. Integrative Genomics Viewer (IGV): high-performance genomics data visualization and exploration. Brief Bioinform. 2013; 14: 178-192.

67. Benelli M, Marseglia G, Nannetti G, Paravidino R, Zara F, Bricarelli FD, Torricelli F, Magi A. A very fast and accurate method for calling aberrations in array-CGH data. Biostatistics. 2010; 11: 515-518. 